\title{
VIA-RAD: a blackboard-based system for diagnostic radiology
}

\author{
Erika Rogers
}

\begin{abstract}
The work described in this article presents an approach to the integration of computerdisplayed radiological images with cooperative computerized assistance for decision-making. The VIA-RAD system (Visual Interaction Assistant for Radiology) is a blackboard-based architecture, founded on extensive data collection and analysis in the domain of diagnostic radiology, together with cognitive modeling of the interaction between perception and problem-solving. The details of this system are presented in terms of domain knowledge representation and domain knowledge mapping. A small prototype of the system has been implemented and tested with radiology subjects, and the results of this study are also described.
\end{abstract}

Keywords: Intelligent systems; Blackboard architecture; Diagnostic radiology; Cooperative decision-making assistance; Perception and problem-solving

\section{Introduction}

The practise of diagnostic radiology is rapidly changing as computerized imaging techniques become more pervasive. While the reading of films is still common in many hospitals, especially with respect to $\mathrm{x}$-rays, the advent of digital acquisition technology for all imaging modalities will eventually make analog representations obsolete. Debates over the inadequacy of spatial and contrast resolutions of digitized $\mathrm{x}$-ray displays compared to those of analog film displays prevail in the literature. However, it is also clear that once a hospital decides to make the 
investment in digital modalities, there is some pressure for the new technology to be used. Moreover, the increasing amount and complexity of electronic data require a more extensive role to be played by computers, a role which ideally combines data, graphic, image and knowledge processing.

The work described in this article presents an approach to the integration of computer-displayed radiological images with cooperative computerized assistance for decision-making. In a visual reasoning task such as diagnostic radiology, that cooperation must be aligned with the cognitive capabilities of the human practitioner. This requires knowledge about perception, about problem-solving, and most importantly, about how perception and problem-solving exchange information in this type of task. By applying cognitive science techniques, a theory of visual interaction has been developed on which the intelligent system architecture is based. The system design itself emphasizes maintaining the human "in the loop". Its goal is to facilitate and stimulate human reasoning capabilities, providing appropriate knowledge-based assistance. Such intelligence consists of knowing what type of assistance is needed and when it may be cognitively useful to afford it. Evaluation of this type of system is based on measuring its effects on the performance of the user.

While current implementations of knowledge-based visualization systems have concentrated primarily on the development of expert-like systems, few are designed to accommodate the 'image' or 'spatial reasoning' that is needed for task domains where the decision-making process must rely on interpretation of a visual image. Systems which do address image reasoning issues may be found primarily in domains that contain sensor-derived data (e.g., $[2,8,14])$. However, even in these systems, the primary intelligence focuses on symbolic decision-making mechanisms, while the choices of image enhancements are left up to the user. This means that the user must understand the effects of the tools, and how to apply them in order to solve the problem.

On the other hand, the approach taken in the work presented here emphasizes understanding something of the underlying cognitive activities during this task, in order to automatically invoke related visualization tools at the appropriate stages in the problem-solving task. This should lead to improved performance by reducing some of the cognitive load (i.e., little or no tool selection needed), and by selecting performance-enhancing rather than simply appearance-enhancing algorithms.

\subsection{Background}

The overall project was divided into four stages of activity: Data Collection and Analysis, Cognitive Modeling of Visual Interaction, System Design and Implementation, and Testing and Evaluation. The first stage began with an examination of how radiologists read and interpret chest $x$-ray images through a number of experiments which culminated in the collection of a number of think-aloud protocols [17]. An encoding scheme was developed which separated the medical concepts from the more general cognitive and descriptive concepts, and this was 


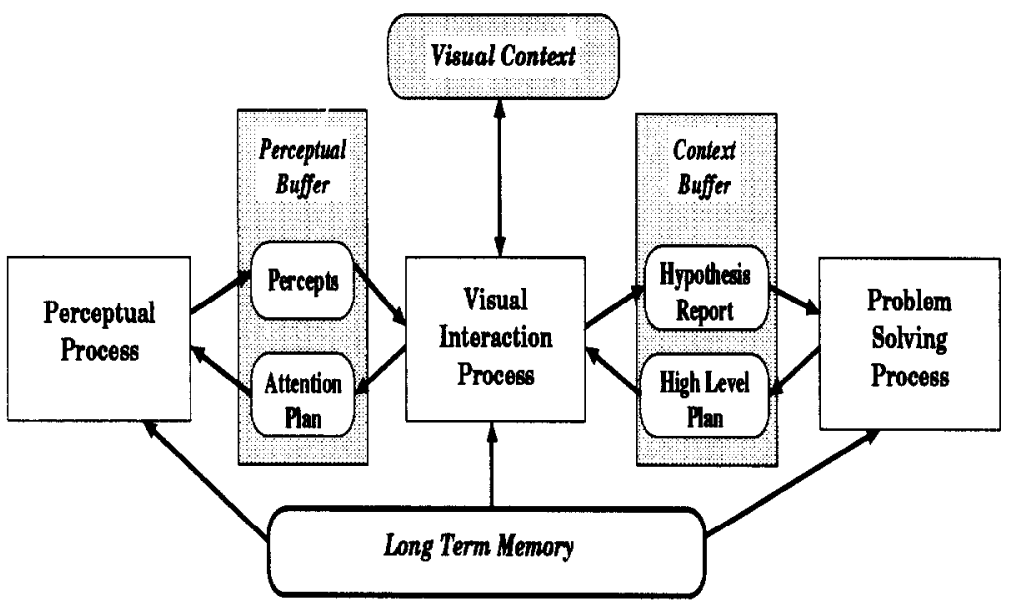

Fig. 1. Cognitive model of visual interaction.

applied to all of the verbal protocols. A detailed decomposition of the data was obtained, which was further analyzed to reveal meaningful contextual patterns [18].

This analysis provided some insights about the task of radiological diagnosis, both in terms of the type of information used, and also how that information is used. Three levels of errors were identified, and several data-driven and expectation-driven reasoning patterns were discerned. The visual information about abnormal objects or findings in the image was expressed in three levels of abstraction: a perceptual level, which had some descriptive value but little diagnostic value; a more general level, which carried some connotations regarding features which might be associated with the object; and, finally, a specialized level, which carried a great deal of diagnostic meaning, and even some expectations about values of associated features. Further results included support for both declarative and procedural context, identification of two particular types of attentional activity (immediate visual capture and deliberate landmark search), and an increased understanding of the role of expectation [19].

All of these empirical results were then used to develop a cognitively-consistent model of the interaction between perception and problem-solving, shown in Fig. 1 [20]. The central idea behind this model is that the information utilized in this type of visual reasoning task is transformed in a number of ways, and that there is a mediating process between perception and problem-solving, called the Visual Interaction Process (VIP), which enables such transformations. The primary functions of the VIP are identified as hypothesis management and attention direction, and the working memory structures which support these activities are described in terms of two conceptual buffers and a visual context store. These symbolic structures represent how working memory may he organized to accommodate the information flow during the task of diagnostic radiology, and it has been shown that the model accounts for empirical results found both in our own data, and in those of Lesgold et al. [9]. 


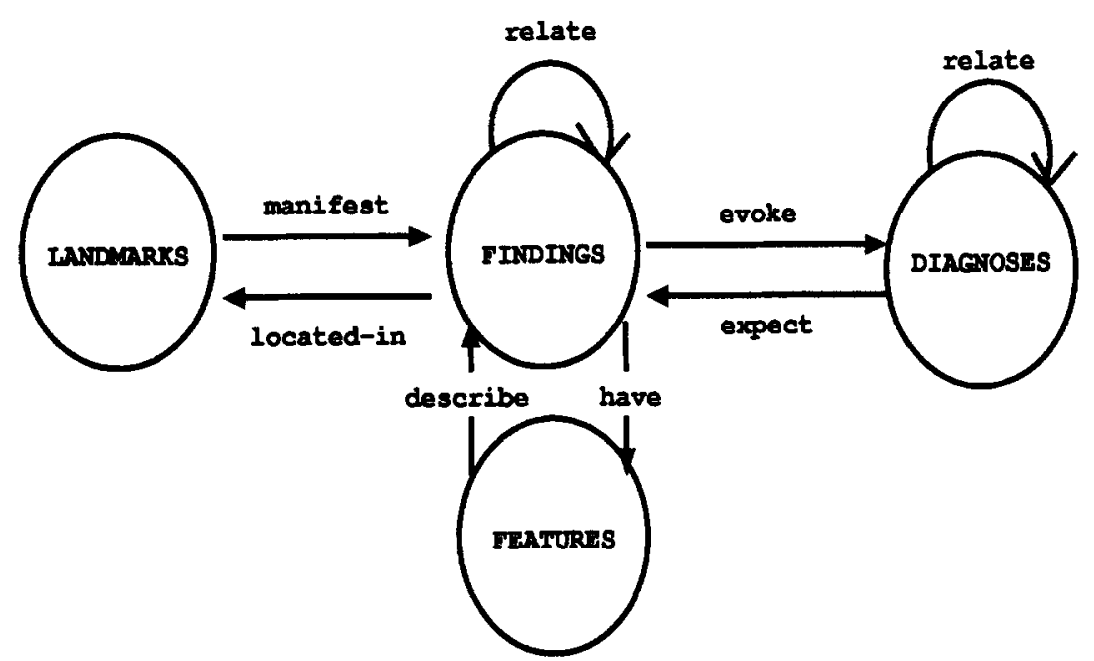

Fig. 2. Relationships between knowledge concepts.

In the third stage of the project, the model was used as the foundation for the design of a knowledge-based computer system called VIA (Visual Interaction Assistant). The VIA system is based on a blackboard-style architecture which incorporates the user, the image display and the program modules into a coherent, cooperative problem-solving system. This article focuses on the third and fourth stages of the project, where the initial architecture is refined into the design of VIA-RAD (Visual Interaction Assistant for Radiology). The implementation and testing of the prototype system is also discussed, together with an evaluation of feedback from radiology subjects.

\section{VIA-RAD knowledge representation}

The task of diagnostic radiology focuses on determining the internal signs of a patient from available radiological images. The full range of information collected on a patient is often not available to the radiologist, with the exception of some case history, and therefore the diagnostic hypotheses are based mainly on image data. The knowledge required for this task consists of four basic components: landmarks, findings, features and diagnoses, and an initial representation of the relationships between these concepts is shown in Fig. 2. While it is typical for diagnostic aid systems to construct their knowledge bases with diagnoses as the basis, the knowledge base of the VIA-RAD system is organized primarily around the findings, with landmarks and diagnoses forming associational related links. This is in keeping with the notion that it is more important for the radiologist to see and understand what is in the image than to force a diagnostic label [23].

In addition to these four knowledge components, the system must also contain information about two important concepts: (1) diagnostic strategies, which will 
direct the gathering of perceptual information in order to converge upon a set of solution hypotheses, and (2) image enhancement algorithms, which can be automatically invoked to support those perceptual activities.

\subsection{Landmarks}

Anatomical objects in the body which are commonly visible or expected to be visible in the image are referred to as landmarks. In this project, the emphasis is placed on chest x-rays, and the landmarks are categorized according to three basic types: aggregate landmarks, which represent collections of individually identifiable objects, individual landmarks, and sub-landmarks or parts. Since all of the anatomical objects described above can be referenced in the process of the chest $\mathrm{x}$-ray diagnosis, they are described by a common knowledge representation, called the Landmark-Frame. The information captured in this representation allows the program to identify the landmark in question, provide mechanisms for navigating the part-of hierarchy, and indicate whether it has been examined by the user, and if so, whether it has been classified as normal or abnormal. In addition, knowledge about spatial relationships between landmarks must be available, and most importantly, knowledge about abnormalities which may occur in that particular landmark must be captured. This last piece of information is the mechanism which allows the program to track the user's data-driven (or bottom-up) diagnostic strategy.

\subsection{Findings}

In the problem-solving literature (e.g., [4]), it is typical for observations of the real world to be considered as data that link up directly with solution hypotheses in the form of triggers or cues. However, in the task of looking at sensor-derived images of the real world, there is uncertainty even at the observation level. The identification of potential or actual abnormalities or findings in an image should therefore be regarded as a first level of hypothesis generation. These finding hypotheses represent a preliminary link between the shadows observed in the $\mathrm{x}$-ray image, and the more abstract concepts that are needed to interpret these observations in the context of the problem-solving task. The three levels of findings discovered in the data analysis stage are expressed as three categories, which correspond to their 'proximity' to either the perceptual or the problem-solving sidc of the model, and are illustrated in Fig. 3.

The Finding-Frame is the knowledge representation which allows the program to identify the particular finding, and to indicate where it lies anatomically. Each finding is described by one or more features such as size, shape, edges, texture, etc., and is associated with other findings as well as particular diagnoses. Since findings are also considered hypotheses, an uncertainty or belief score is also included that indicates the strength of the hypothesis. An example of a general finding frame is illustrated in Fig. 4. 


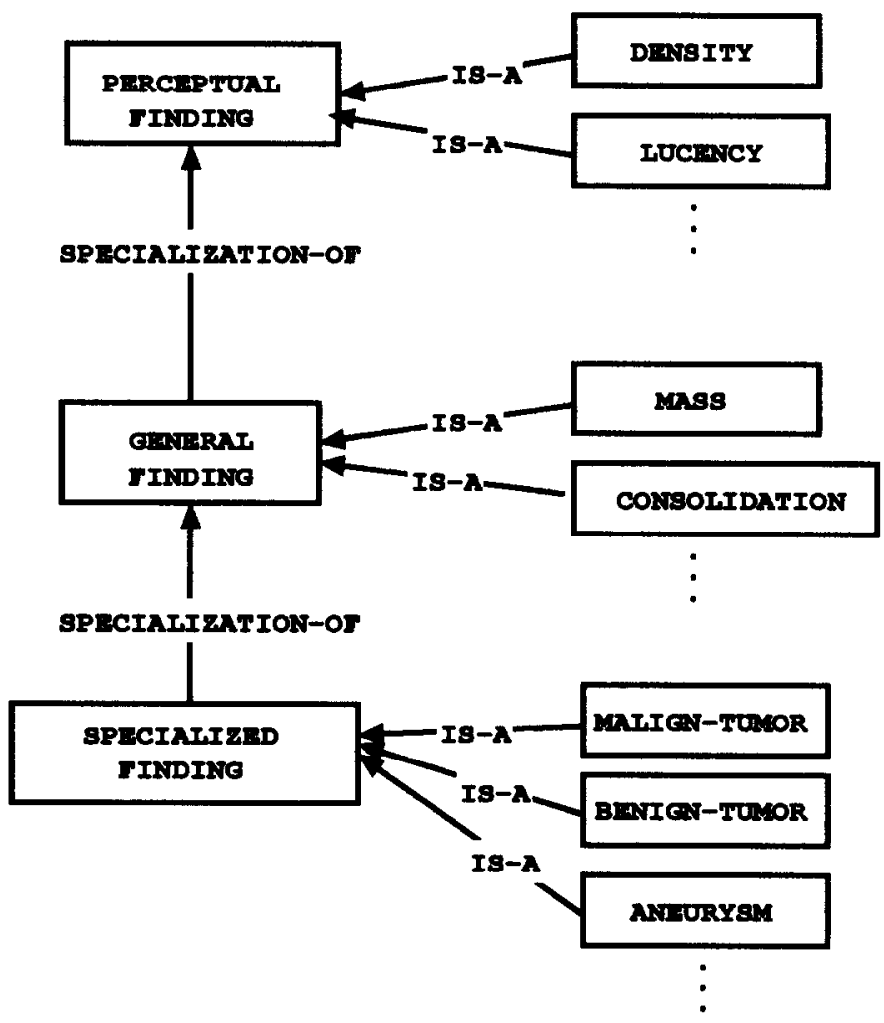

Fig. 3. Findings hierarchy.

\begin{tabular}{|r||l|}
\hline \multicolumn{2}{|c|}{ Mass Finding Frame } \\
\hline NAME & mass \\
\hline FINDING-TYPE & general \\
\hline LANDMARK-LOC & chest \\
\hline MUST-HAVE & no-features \\
\hline MUST-NOT-HAVE & no-features \\
\hline SPECIALIZATION-OF & no-findings \\
\hline GENERALIZATION-OF & $\begin{array}{l}\text { (adenopathy, aneurysm, benign-tumor, } \\
\text { malignant-tumor, granuloma, nodule, } \\
\text { other, unknown) }\end{array}$ \\
\hline TRIGGERS & no-diagnoses \\
\hline SUPPORTS & no-diagnoses \\
\hline CONTRADICTS & $\begin{array}{l}\text { no-diagnosis } \\
\text { undefined }\end{array}$ \\
\hline
\end{tabular}

Fig. 4. Example general finding frame. 


\subsection{Features}

Features are concepts that are used to describe the findings in an image, and can contribute visual evidence which will support or rule-out a finding hypothesis. Our experimental results reveal a small number of features that are important in both directions of the diagnostic reasoning process (data-driven and expectationdriven). In data-driven reasoning, the value of a feature may lead to the evocation of a correct finding hypothesis (or set of hypotheses), and further, to a correct diagnosis hypothesis. On the other hand, if a particular diagnosis hypothesis is currently active, it may expect a certain value for a feature, which, when determined, may affect belief in that diagnosis. However, features are also most dependent upon the quality of the image under consideration, and the perceptual talents of the radiologist. Therefore, the Feature-Frame representation also contains procedural attachments for invoking appropriate image enhancements, as well as slots for expected and observed values of the features. (These latter are qualitative in nature, rather than statistical.)

\subsection{Diagnoses}

The traditional approach of automated reasoning programs in the medical domain has been to consider the determination of disease as the solution to the diagnostic problem. Researchers such as Patil have described different levels of hypotheses used by physicians to do diagnosis [15]. INTERNIST-1 [11] had individual diseases as the building blocks for its knowledge base, and first explored the use of a definitional or inheritance hierarchy of diagnostic hypotheses. This approach has also been used in the design of RADIO [6], an expert system for radiological diagnosis. MDX [1], on the other hand, considered the relationship between diseases and findings to be evidential or associational, and this approach was found to be effective in reducing the number of hypotheses.

In the VIA-RAD project, however, a greater emphasis is placed on the effective collection of image findings, rather than on the absolute determination of a disease diagnosis. Therefore, diseases are organized simply as a collection of diagnostic hypotheses, which are primarily categorized as malignant or benign. The knowledge represented in the Diagnosis-Frame structure emphasizes the associations between the diagnoses and findings, and also the information needed to calculate the belief value of the diagnostic hypotheses. Examples of models to handle uncertainty in medical AI systems include the certainty-factor model of MYCIN [21], the evoking-strength/frequency-weight model of INTERNIST-1 [11], and the causal-weighting model of CASNET [25]. Shortliffe, in his review of clinical decision-making [22], also discusses the resurgence of interest in probabilistic methods to handle uncertainty. Belief management in the VIA-RAD system must incorporate a number of aspects, including the user's confidence in the triggering finding, evoking strength of the triggering finding, frequency of the diagnosis, and the user's belief in the diagnosis. This is then modified by the discovery of evidence for or against the diagnosis. Due to the limited availability of adequate information 
on likelihoods and appropriate weighting values, a simple ad hoc model was adopted in the prototype system, which placed greater emphasis on the user's confidence and belief input. Although this proved adequate for initial testing of system performance, a more sophisticated belief management method must be adopted in future versions.

\subsection{Diagnostic strategies}

In medical diagnosis, strategies most often are associated with ways of reasoning about a patient, given a particular set of inputs, and the current state of the solution space. Hypothetico-deduction is a strategy well-known in the literature of medical diagnosis (e.g., [10]) which is used to generate hypotheses from a set of input data. The use of abductive inference (e.g., [4]) to converge on a parsimonious set of diagnoses leads to further strategies for managing hypotheses once they have been generated. One such strategy is to try to rule out hypotheses by seeking evidence that differentiates between the choices. The search for a 'best explanation' leads to a strategy in which supporting evidence is sought for the best candidate hypotheses. Another strategy is to emphasize the gathering of evidence because there is insufficient information to generate or strongly support a set of candidate hypotheses. The four diagnostic strategies which Mutalik et al. [12] identified in their expert critiquing system for aiding radiological diagnosis form the basis for VIA-RAD's collection of strategies: Pursue, Rule-In-Rule-Out, Not-Enough-Information and Conflict. This list is extended to also include Deliberate Landmark Search, which appears to be commonly used when there is no immediate abnormality which captures the radiologist's attention. The prototype system focused primarily on the first three of these strategies.

\subsection{Image enhancements}

The visibility of landmarks, findings and features is critical to the formulation of diagnostic hypotheses, and therefore the utility of image enhancements is most intimately tied to these three categories. To date, solutions to particular image enhancement problems have concentrated on limited types of abnormalities, and there has been little work done on integrating numerous types of image enhancements into a flexible diagnostic system. Systems which have attempted such integration usually provide a toolbox from which the radiologist must choose an appropriate technique to apply. This approach has major drawbacks in a clinical setting. Early work by Tuddenham on $\mathrm{x}$-ray diagnosis presented a rather pessimistic outlook on the efficacy of image enhancements to improve diagnostic performance [24]. However, more recent research suggests that there are a number of techniques now available which do positively impact radiological diagnostic performance to some extent, especially with respect to detection tasks. A detailed discussion of some relevant work on image enhancements and its impact on the development of a cooperative diagnostic radiological assistant is presented in [19].

Due to system limitations, the VIA-RAD prototype only contained a small number of image enhancements, including histogram equalization, which is a 
well-known technique for contrast enhancement [7], and inverse image, which was incorporated in accordance with the results of Coristine et al. [3]. Further work on the VIA-RAD project will include edge enhancements such as the nonlinear masked sifting methods developed by Herman [5], and region of interest selection, allowing local enhancement application while maintaining the surrounding image context [16].

\section{VIA-RAD knowledge mapping}

To assist and enhance the diagnostic visual interaction process, the VIA system incorporates both domain knowledge and process knowledge, and is conceptualized as an overlay to the user's own visual interaction process. At critical stages, the system affords directions to the user to focus attention on particular aspects of the problem, either in the hypothesis space or in the image itself, and provides appropriately enhanced information. In order to do this, the system is designed to retrieve, combine and post knowledge in a manner that is consistent with the cognitive model of visual interaction. The overall VIA-RAD system design is presented in Fig. 5, and the following subsections describe the main components.

\subsection{VLA-RAD blackboard}

The VIA-RAD blackboard represents working memory, and it is where the evolutionary results of the problem-solving effort are captured. The buffers of the underlying cognitive model suggest a logical partitioning of the blackboard that facilitates the corresponding types of information transfer. The general VIA blackboard consists of a Context area, a Perceptual area, an Hypothesis area and an Attention area, and these are customized in the VIA-RAD system to reflect the specific needs of the radiological domain.

The Context area contains information that is known about the overall problem context. Typically, the image will contain expected objects in particular configurations that are considered standard or normal. The presence or absence of such objects, and their normal or abnormal classification constitute contextual events, which must be posted to the blackboard. In the VIA-RAD system, this is represented by the Landmarks Panel. The Perceptual area, on the other hand, is where the system posts the dynamic perceptual input about what is in the image. This information, obtained primarily from the user, is then used by the knowledge sources of the system to formulate hypotheses. The corresponding VIA-RAD structure is the Features Panel.

Current hypotheses that constitute the partial (or complete) solutions that are evolving as a result of the problem-solving activity are contained in the Hypothesis area. This region is further divided into two sections, corresponding to the two types of hypotheses that must be considered: Visual Hypotheses, which reflect what is currently known about abnormal or unexpected objects in the image, and Reasoning Hypotheses, which correspond to explanations of those objects, or 


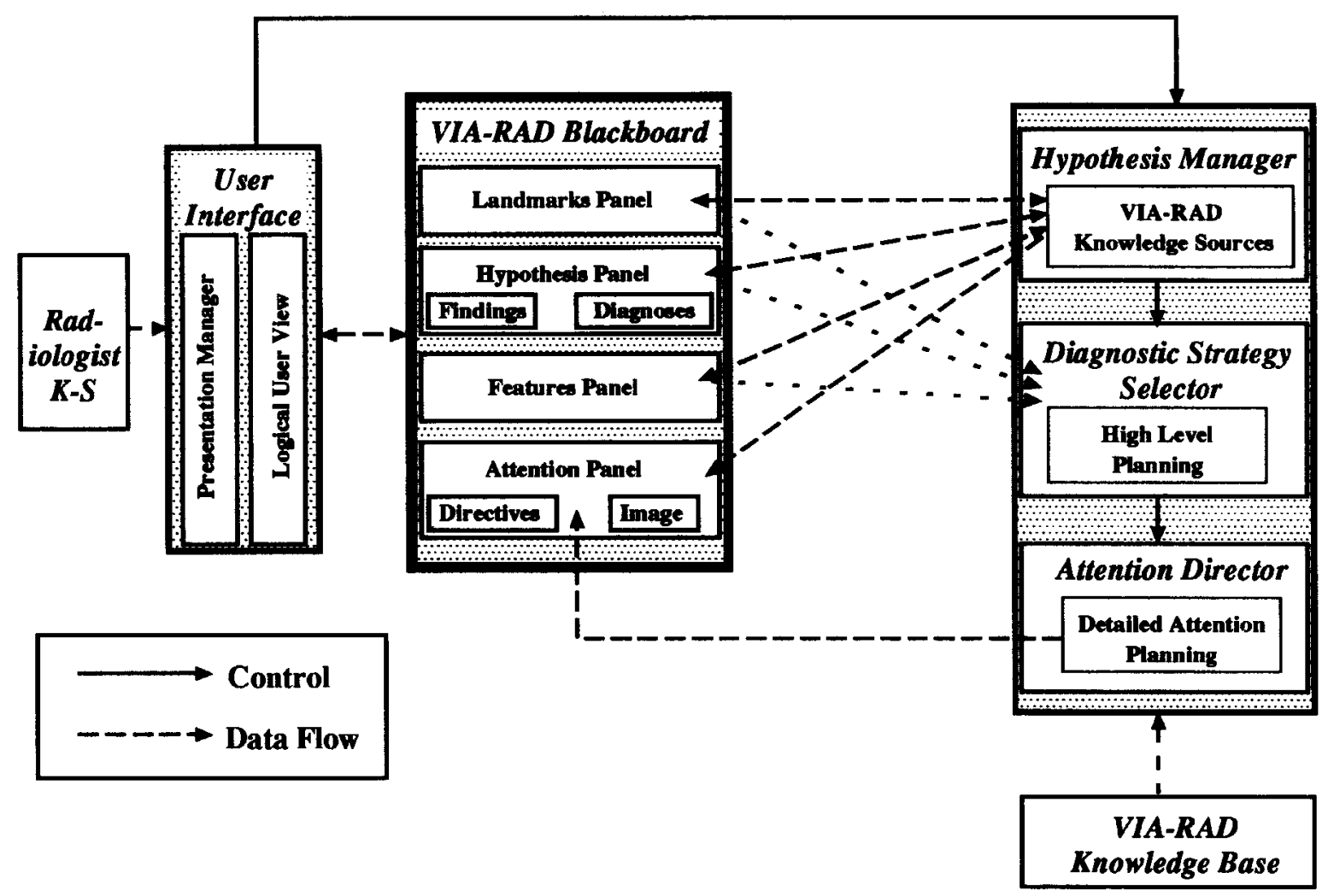

Fig. 5. VIA-RAD system design. 
collections of objects. In the VIA-RAD system, the former is represented by the Findings portion of the Hypothesis Panel, while the latter refers to the Diagnoses portion.

The Attention Panel is the locus of the visual focus-of-attention mechanism. It also is partitioned into two subpanels. The first contains attention directives aimed at the perceptual process of the user, and thus reflects the Attention Plan part of the model. The second subpanel is used to display the images (original and enhanced). The image is incorporated into the blackboard because it, too, is a dynamic part of the problem-solving activity. The system directs the user's attention not only by textual suggestions, hut by executing appropriate image enhancements designed to facilitate the user's perception of the feature in question.

\subsection{Contrul}

The VIA system has a control structure that is primarily based on the functions of the Visual Interaction Process (VIP) of the model. It has been suggested that the VIP, in its role as mediating process, impacts the problem-solving process through hypothesis management, and the perceptual process through control of the focus of attention mechanism. Therefore two of the main control modules of the VIA system reflect this functionality, and are called the Hypothesis Manager, and the Attention Director. The former impacts the blackboard through the activities of hypothesis-related knowledge sources, while the latter is concerned with focusing attention by altering the image and/or presenting suggestions to the user of what to look at next. In order to pass control from the Hypothesis Manager to the Attention Director, a third control module is needed, which is called the Strategy Selector. Since there may be several ways in which attention could be focused in order to obtain perceptual information, the Strategy Selector makes this decision, based on current blackboard information.

One of the important aspects of the design of VIA is that the user is conceptualized as an independent knowledge source in the system. As such, he or she reads from and writes to the blackboard in an effort to cooperatively solve the problem that normally would be done by the user alone. Therefore the VIA system has a somewhat looser control mechanism than is common for typical blackboard systems. Unlike systems which perform automatic reasoning, based on streams of input data (e.g., HASP/SIAP, Tricero, Crysalis [13]), VIA depends, to a great extent, on the user for input, particularly of a perceptual kind. The user's actions can only be interpreted in terms of how well VIA can fit them into the underlying model of visual interaction. The system, however, does not control the user's responses - it only suggests and attempts to guide the problem-solving along certain directions, and prompts the user for feedback. Communication between the user and the rest of the VIA system is therefore an important consideration, and takes place through the User Interface, the fourth control module. It is divided into two parts: the Logical User View, which controls how much of the blackboard is visible to the user, and the Presentation Manager, which controls the form of the interface as it is presented to the user. 


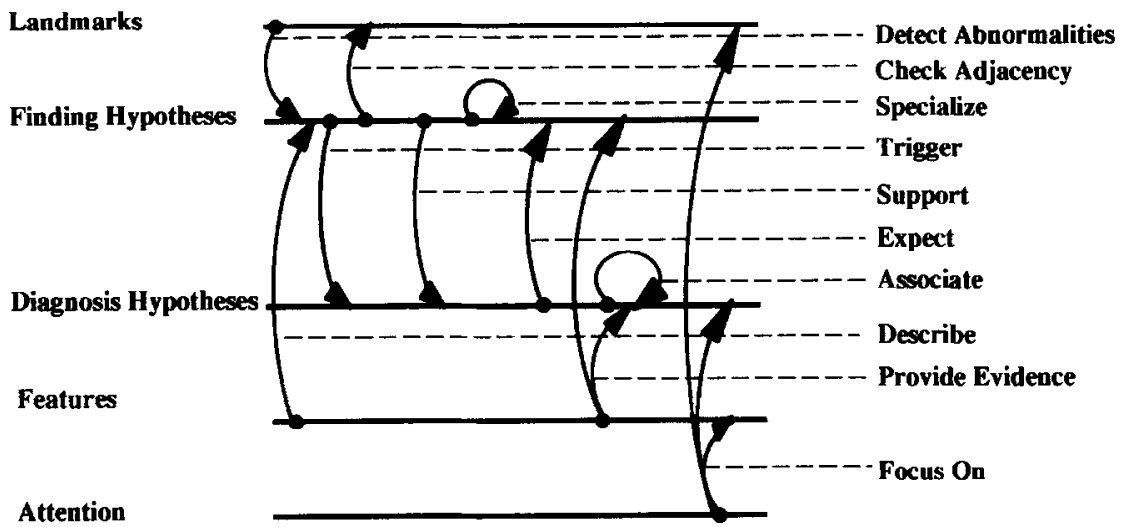

Fig. 6. Blackboard levels and knowledge sources.

\subsection{Knowledge sources}

The knowledge sources of VIA-RAD have preconditions which, when satisfied, alter the information at one or more of the other levels of the blackboard. These relationships are shown in Fig. 6, in a form adapted from the Hearsay-II literature [13]. In the initial implementation, a simple agenda control mechanism has been adopted where the preconditions of the appropriate knowledge sources are matched to the blackboard panel most recently modified by the user. If more than one knowledge source is satisfied, they are sequentially activated and the user must wait until all affected blackboard panels have been updated before proceeding. This approach is adequate for the small number of knowledge sources currently identified, since there is not a great deal of competition, and there is some inherent ordering in the knowledge sources themselves. If a problem-solving strategy has been invoked, this further restricts the knowledge sources which can operate at that time. Fig. 7 shows an example of a hypothetical interaction with the VIA-RAD system, where a knowledge source is activated, a strategy is invoked, and attention is directed for further problem-solving.

\section{Testing and evaluation}

The prototype VIA-RAD system was implemented on a Pixar/Sun Workstation computer system (the same one used to collect the original protocol data), and the scope of the program was restricted to a small number of cases which contained only mass abnormalities, both benign and malignant. The main purpose of this effort was to demonstrate the capabilities of the system with respect to the activation of the knowledge sources, the invocation of strategies, and the subsequent adjustment of belief values. 


\section{User View of}

\section{VIA-RAD Blackboard}

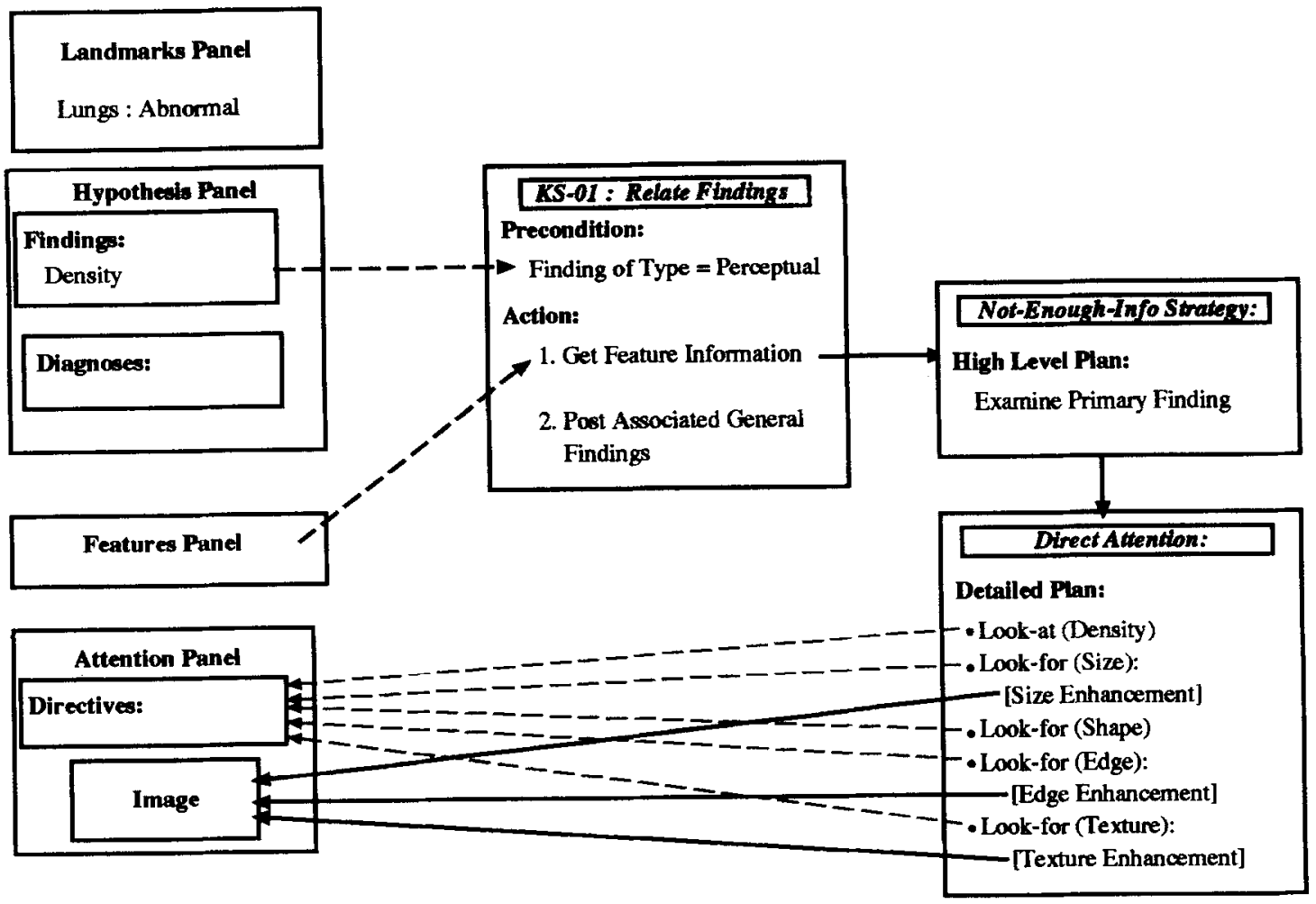

Fig. 7. VIA-RAD example interaction. This shows the user's view of the blackboard on the left hand side, and the system-invoked activity on the right hand side. Here initial information posted by the user satisfies the precondition of the Relate Findings Knowledge Source (KS-01). This KS requires more perceptual information before it can complete its actions, and therefore it invokes the Not-Enough-Information strategy. The High Level Plan formulated as a result of the strategy leads to a Detailed Attention Plan, which modifies the Attention Panel by posting instructions to the user, and enhancing the image.

The display of the VIA-RAD prototype consisted of two computer screens: one with the non-interactive display of the digitized chest $x$-ray images, and the other with a simple window display of the blackboard panels. An example screen in shown in Fig. 8. Due to the highly interactive nature of the VIA-RAD system, it was decided that user feedback would be very important, even for early versions of the prototype, and therefore, an observational study was designed to test the system.

\subsection{Subjects and materials}

Five subjects were recruited from the Emory University Radiology Residency Program and ranged in experience from second to fourth year residents. None of 


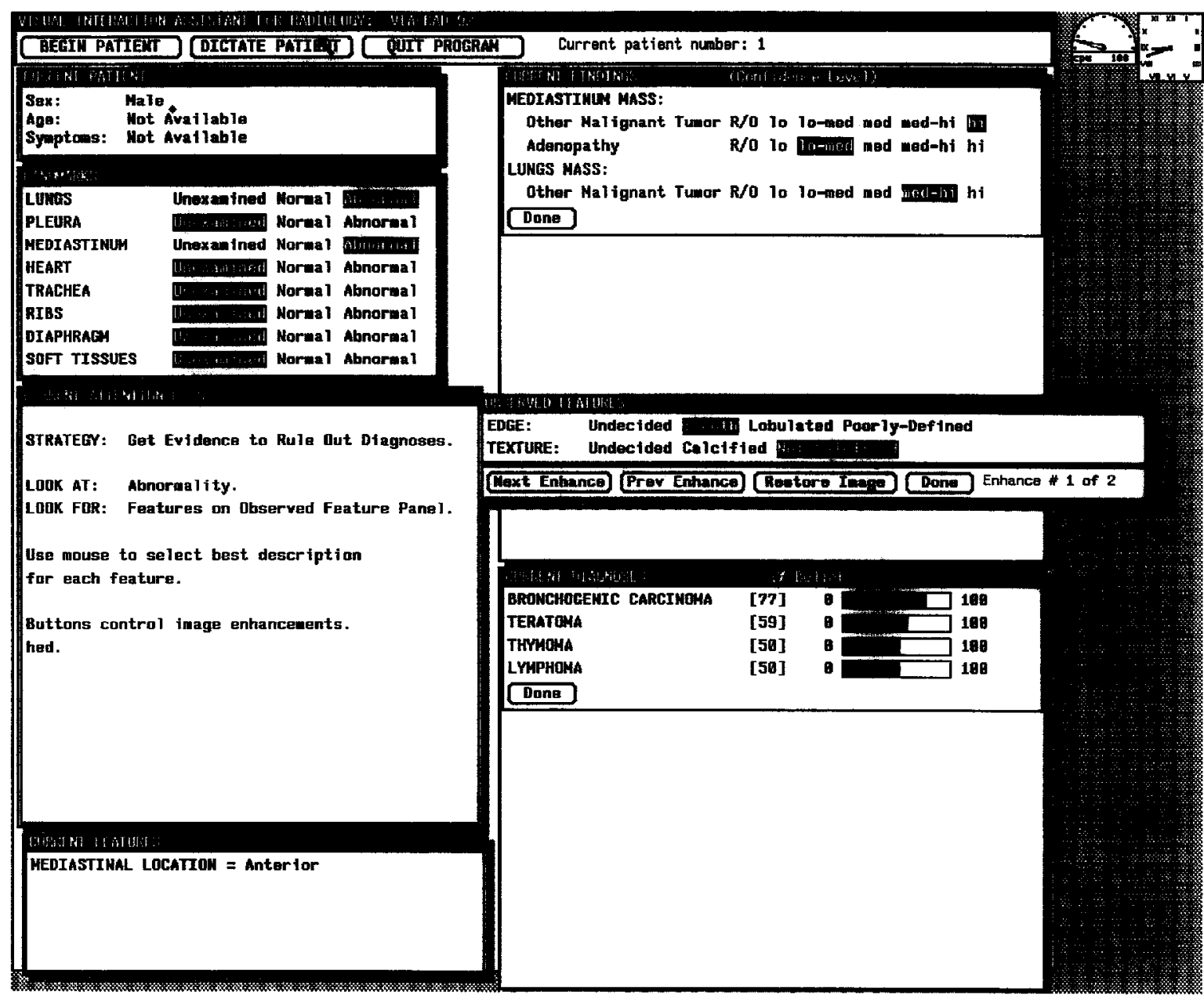

Fig. 8. Sample screen layout. 
the subjects had been involved in any of the previous studies related to this project. Four cases, representing benign and malignant tumors in the lung and mediastinum, were selected for the experiment, and digitized images as well as corresponding dictations by the participating medical expert were prepared. Written materials included a Consent Form, Orientation Instructions, and a rating sheet was also provided to collect information for each case on size estimates, image quality, confidence in diagnosis, and case difficulty. In addition, a post-session questionnaire was used to obtain retrospective feedback from the subjects regarding their reactions to the program.

\subsection{Methodology}

The overall scheme of the study was to have the subjects read two cases from the digitized display as they normally would, and then to read the last two cases using the VIA-RAD system. The cases were varied from subject to subject so that data from both reading methods (with VIA-RAD and without) was available for each case. For the first part of the study, the subjects were asked to examine the two digitized chest x-ray cases, and then to dictate them. After each dictation, a static VIA-RAD-like rating screen was displayed, and the subject was asked to fill in this 'form' according to what was in the current image. When this form was completed, the system stored a snapshot image of the screen, and the subject was then asked to rate the case with respect to image quality, confidence in diagnosis and case difficulty. A size estimate was also requested, if appropriate. The second part of the study consisted of the subject interacting completely with the VIA-RAD system in order to diagnose the remaining two digitized chest $\mathrm{x}$-rays. They were free to ask questions at any time, regarding system behavior, and indeed, it was often necessary to inform them that something was about to occur, due to the slow response of the VIA-RAD window interface. The entire session was audiotape recorded, and the post-session questionnaire was administered to ascertain the subject's experience level and free-response to the VIA-RAD system. The data collected for this observational study included transcriptions of the recorded dictations, the ratings forms, trace files of the subjects' interaction with the computer system, and screendumps of their final screens, from both parts of the testing sessions.

\subsection{Analysis and results}

The ratings for image quality, confidence and case difficulty were tabulated for all subjects and all cases, and the aggregated results are shown in Table 1. These results indicate that, in general, subjects gave more positive ratings when they were not using the VIA-RAD system. Image quality was rated better, confidence was rated higher, and cases were rated somewhat easier without VIA-RAD. A further analysis was undertaken to examine the overall performance trends based on a comparison of the final dictation of the project expert and the subjects' dictations. A score of zero (0) was assigned if the subject did not mention the same primary 
Table 1

Aggregate ratings for all subjects and all cases. Scores range from 1 to 5

\begin{tabular}{lll}
\hline & With VIA-RAD & Without VIA-RAD \\
\hline Image quality & 2.9 & 3.7 \\
Confidence & 3.2 & 3.6 \\
Case difficulty & 2.7 & 2.3 \\
\hline
\end{tabular}

For image quality, $1=$ not adequate, $3=$ adequate, and $5=$ very good. For confidence rating, $1=$ low, $3=$ medium, and $5=$ high. For case difficulty, $1=$ easy, $3=$ challenging, and $5=$ difficult.

diagnosis anywhere in the dictation. A score of one (1) was assigned if a similar word was used, but not the precise expression used by the project expert (e.g., cancer versus bronchogenic carcinoma), and, finally, a score of two (2) was given if the subject used the same expression as the expert. Some cases had important secondary features or diagnoses mentioned by the expert, and these were scored in the same way as above. These results are also presented in aggregate form in Table 2. (A formal statistical analysis was not conducted at this time.) This analysis shows a considerable difference in diagnostic performance between the instances where VIA-RAD was used, compared to those where it was not. These differences could be due to the fact that the subjects spent more time on the cases where they had to use VIA-RAD, and therefore produced better diagnostic reports. In addition, the presentation of the VIA-RAD selections may have influenced the vocabulary used by the subjects to dictate their reports. This latter condition, however, was somewhat alleviated by the fact that at the end of the first non-VIA-RAD reading, the subjects were presented with the static information screen to be filled in. Given the limitations of the testing conditions, including a small subject population, a limited sample of test cases, and no time to accommodate learning effects, it is not possible to draw definitive conclusions about whether the positive performance results were due to the effectiveness of the VIA-RAD system itself, or were just fortuitous side effects.

It is interesting to note, however, that the overall ratings given by the subjects show that both image quality and confidence in diagnosis were higher without VIA-RAD, and that cases were, on average, considered to be less difficult. There are a number of possible explanations for this - perhaps the use of an unfamiliar computer system caused the subjects to feel somewhat more insecure when rating the cases. On the other hand, this does not explain the relatively poor performance when subjects did not use the VIA-RAD system. It is possible that there is some over-confidence in the diagnostic readings that is not always borne out in accuracy.

Table 2

Performance across all subjects and all cases. Overall percent matching project expert's diagnosis

\begin{tabular}{lll}
\hline & With VIA-RAD & Without VIA-RAD \\
\hline Primary & $81 \%$ & $25 \%$ \\
Secondary & $67 \%$ & $33 \%$ \\
\hline
\end{tabular}


From the post-session questionnaires, subjective reactions to the program were obtained, which were, in general, quite positive. The limited knowledge base and slow response of the system were most often cited as hindrances. On the other hand, the subjects mentioned both image enhancements and the presentation of candidate hypotheses as helpful features. It was felt that the program often presented findings and/or diagnoses which should have been considered, but might otherwise have been overlooked. Based on the overall results obtained from this study, it is felt that the preliminary work has shown sufficient promise to pursue further development.

\section{Conclusion}

The work presented in this article was inspired by an interest in how visual information is used by human practitioners to solve diagnostic problems. New developments in visual computer displays have opened up extensive possibilities for the use of computers as tools in such endeavors. However, current hardware and support software have improved much more rapidly than applications software which can fully utilize this power. On the other hand, despite technology's advances, there are many areas where it is acknowledged that human decisionmaking capabilities are still far superior to those of the machines, and are likely to be so for some time to come. There is, therefore, a great deal of incentive to design computer systems which can cooperate with humans in the execution of such complex tasks.

It has been said, with some concern, that this type of system may change the way that radiologists perform their task. This is true, and an argument in favor of such change is that, with the introduction of more and more new technology for image acquisition, radiologists are already being forced to change and to accommodate new ways of viewing patient data. Therefore, the vision for technology in this field must reach beyond acquisition, storage and retrieval issues. With the advent of totally digital radiology departments, software systems should be provided which will enable the radiologists to take advantage of the coupling of computational power with cognitive abilities to produce an effective decision-making environment. This will lead eventually to a much higher quality of patient care and management.

\section{References}

[1] B. Chandrasekaran, S. Mittal and J.W. Smith, Radex - towards a computer-based radiology consultant, in: E.S. Gelsema and L.N. Kanal, eds., Pattern Recognition in Practice (North Holland, Amsterdam, 1980) 463-477.

[2] A.I. Cohn, P.L. Miller, P.R. Fisher, P.G. Mutalik and H.A. Swett, Knowledge-based radiologic image retrieval using axes of clinical relevance, Computers Biomed. Res. 23 (1990) 199-221.

[3] M. Coristine, M. Goldberg, C. Beeton, R.F. Dillon, J.W. Tombaugh, G. Belanger and J. Ahuja, The use of IRIS image enhancement facilities on digital images by radiologists during a clinical 
trial at the Ottawa Civic Hospital, SPIE Medical Imaging IV: Image Processing 1233 (1990) 426-438.

[4] O. Fischer, A. Goel, J.R. Svirbely and J.W. Smith, The role of essential explanation in abduction, Artificial Intelligence in Med. 3 (1991) 181-191.

[5] S. Herman, Feature-size dependent selective edge enhancement of $\mathrm{x}$-ray images. SPIE Medical Imaging II 914 (1988) 654-659.

[6] W. Horn, H. Imhof, B. Pfahringer and E. Salomonowitz, A radiological expert system for the PC - design and implementation issues, in: J. Fox, M. Fieschi and R. Engelbrecht, eds., Proc. European Conference on AI in Med. (AIME) 1987 (Springer-Verlag, Bcrlin, 1987) 169-176.

[7] A.K. Jain, Fundamentals of Digital Image Processing (Prentice Hall, Englewood Cliffs, NJ, 1989).

[8] K.-F. Kraiss, Knowledge-based classification with interactive graphics, in: E. Hollnagel, G. Mancini and D.D. Woods, eds., Intelligent Decision Support in Process Environments (Springer-Verlag, Berlin, 1985) 363-369.

[9] A. Lesgold, P. Feltovich, R. Glaser and Y. Wang, The Acquisition of Perceptual Diagnostic Skill in Radiology, LRDC Technical Report No. PDS-1, 1981.

[10] W. McGaghie, Medical problem-solving: a reanalysis, J. Med. Education 55 (1980) 912-921.

[11] R. Miller, H. Pople, Jr. and J. Myers, INTERNIST-1, an experimental computer-based diagnostic consultant for general internal medicine, New Engl. J. Med. 307 (1982) 468-476.

[12] P. Mutalik, P. Fisher, H.A. Swett and P.L. Miller, Structuring coherent explanation: the use of diagnostic strategies in an expert critiquing system, Proc. 12th Annu. Symp. Computer Applications in Med. (IEEE, Washington, DC, 1988) 26-31.

[13] H.P. Nii, Blackboard systems: blackboard application systems, blackboard systems from a knowledge engineering perspective, AI Mag. (August 1986) 82-106.

[14] $\mathrm{K}$. Ohe and S. Kaihara, Intelligent atlas: a method to support physicians' spatial reasoning, MEDINFO 89, Proc. 6th Conference on Medical Informatics (North-Holland, Amsterdam, 1989) 175-179.

[15] R.S. Patil, Artificial intelligence techniques in medical diagnosis, in: H.E. Shrobe and AAAI, eds., Exploring Artificial Intelligence (Kaufmann, San Mateo, CA, 1988) 347-379.

[16] G. Revesz and H. Kundel, Psychophysical studies of detection errors in chest radiology, Radiology 123 (1977) 559-562.

[17] E. Rogers, R.C. Arkin, M. Baron, N. Ezquerra and E. Garcia, Visual protocol collection for the enhancement of the radiological diagnostic process, Proc. First Conference on Visualization in Biomedical Computing (IEEE, Los Alamitos, CA, 1990) 208-215.

[18] E. Rogers, R.C. Arkin and M. Baron, Visual interaction in diagnostic radiology, Computer-Based Medical Systems, Proc. Fourth Annu. IEEE Symposium (IEEE, Los Alamitos, CA, 1991) 170-177.

[19] E. Rogers, Visual Interaction: a Link Between Perception and Problem-Solving, Ph.D. Dissertation, Georgia Institute of Technology, Tech Report No. GIT-CC-92/59, Atlanta, GA, 1992.

[20] E. Rogers, A cognitive theory of visual interaction, in: B. Chandrasekaran, J. Glasgow and N.H. Narayanan, eds., Diagrammatic Reasoning: Computational and Cognitive Perspectives (AAAI/MIT Press, forthcoming).

[21] E.H. Shortliffe, Computer-based medical consuliations: MYCIN (Elsevier, New York, 1976).

[22] E.H. Shortliffe, Medical informatics and clinical decision making: the science and the pragmatics, Medical Informatics and Clinical Decision Making 11 (4) (1991) Supplement S2-S14.

[23] L.F. Squire and R.A. Novelline, Fundamentals of Radiology (Harvard University Press, Cambridge, MA, 1988).

[24] W. Tuddenham, Visual search, image organization, and reader error in roentgen diagnosis, Radiology 78 (1962) 694-704.

[25] S. Weiss, C. Kulikowski and A. Safir, A model-based consultation system for the long-term management of glaucoma, IJCAI 5 (1977) 826-832. 